\title{
¿Es posible la felicidad en estudiantes universitarios?: un estudio no paramétrico
}

\author{
Bernadine Leticia Dixon Carlos ${ }^{1}$, llenia Arllery García Peralta ${ }^{2}$ \\ Sandra Carolina Rojas Hooker ${ }^{3}$, Tania Patricia Rossmann Hooker ${ }^{4}$ \\ Elebe Williams Muller ${ }^{5}$, Víctor Ronald Zúniga Morales ${ }^{6}$
}

\section{Información de artículo:}

Recibido: 30/09/2019

Aprobado:30/10/2019

\section{Palabras claves:}

alegría,

etnia,

felicidad,

género,

realización,

satisfacción.

\section{Resumen}

La pregunta ¿es posible la felicidad en estudiantes universitarios? guía la presente investigación cuantitativa dirigida a 237 estudiantes (hombres y mujeres) de la Universidad de las Regiones Autónomas de la Costa Caribe Nicaragüense. Investigación que está sustentada en un diseño descriptivo, aplicando la escala de felicidad de Alarcón (2006) tomando en consideración las variables de género, edad, etnia y años de estudio. Para ello, se realizó diferentes tipos de análisis, entre estos: de consistencia interna del cuestionario, el análisis de componentes principales, y un estudio de correlaciones entre las categorías aplicando las pruebas de normalidad de Kolmogorov, la prueba U de Mann-Whitney, prueba de Wilcoxon, Kruskal Wallis, y la prueba de Friedman, utilizando el paquete estadístico de SPSS. Los resultados demuestran que la felicidad en estudiantes universitarios sí es posible tomando en cuenta que la misma es meramente subjetiva en las personas y, a su alrededor se engloban diferentes elementos que la hacen posible. En este sentido, se concluye que las variables etnia, edad, género y años de estudio no tienen una relación directa con el estado de felicidad de los estudiantes, pues no son determinantes para definir este estado en el ser humano.

\footnotetext{
${ }^{1}$ Máster en Género y Desarrollo. Directora del Centro de Información de la Mujer de la Universidad de las Regiones Autónomas de la Costa Caribe Nicaragüense. Email: bernadixonc@gmail.com, (1): https://orcid.org/oooo-ooo3-3503-6516

2 Máster en Gerencia Estratégica. Coordinadora de Cooperación Externa de la Universidad de las Regiones Autónomas de la Costa Caribe Nicaragüense- Recinto Universitario Bluefields. Email: ilenia.garcia@uraccan.edu.ni, (1): https://orcid. org/oooo-0oo2-6152-8779

3 Máster en Estudios Indígenas. Directora del Instituto de Estudios y Promoción de la Autonomía de la Universidad de las Regiones Autónomas de la Costa Caribe Nicaragüense - Recinto Universitario Bilwi. Email: srojashooker@gmail.com, (1): https://orcid.org/oooo-0002-7467-2366

4 Máster en Derechos Humanos y Democracia. Oficial de Programa de la Universidad de las Regiones Autónomas de la Costa Caribe Nicaragüense. Email: tania.rossman@uraccan.edu.ni, (1): https://orcid.org/oooo-ooo2-4627-2410

5 Máster en Estadística Computacional. Profesor de la Universidad de las Regiones Autónomas de la Costa Caribe Nicaragüense - Recinto Universitario Bilwi. Email: elebewilliams@gmail.com, (1): https://orcid.org/oooo-ooo2-5238-6481

${ }^{6}$ Máster en Desarrollo Rural con Enfoque Territorial. Director de Innovación y Emprendimiento de la Universidad de las Regiones Autónomas de la Costa Caribe Nicaragüense Email: victor.zuniga@uraccan.edu.ni, (1): https://orcid.org/oooo-0002-4063-2219
} 


\section{Is happiness in University student possible? A non-parametric study}

\section{Abstract}

The question, Is happiness possible in university students? guides the present quantitative research aimed at 237 students (men and women) of the University of the Autonomous Regions of the Nicaraguan Caribbean Coast. This Research is based on a descriptive design, applying the Alarcon's scale of happiness (2006) taking into account the variables of gender, age, ethnicity and years of study. For this, different types of analysis were performed, among them: internal consistency of the questionnaire, the analysis of main components, and a study of correlations between the categories applying the Kolmogorov normality tests, the Mann-Whitney U test, test from Wilcoxon, Kruskal Wallis, and the Friedman test, using the SPSS statistical package. The results show that happiness in university students is possible taking into account that it is merely subjective in people and, around it, different elements are included that make it possible. In this sense, it is concluded that the variables ethnicity, age, gender and years of study do not have a direct relationship with the state of happiness of the students, since they are not determinants to define this state in the human being.

Key Words: ethnicity, fulfillment, gender, happiness, joy, satisfaction

\section{Introducción}

¿Qué es la felicidad? una pregunta que todas y todos nos hemos hecho alguna vez en la vida y que preocupa tanto a la sociedad que hasta existen diversas escalas para evaluar el bienestar, que se asocian a la evaluación de la felicidad a nivel teórico, así como en su contenido temático o en sus dimensiones. Según Alarcón (2006), la felicidad se evalúa en función al estado afectivo y cognitivo, por medio del cual se evalúan aspectos positivos y negativos, emitiendo un juicio global acerca de la satisfacción con la vida, por ende, se emite una conducta feliz.

El estudio de la felicidad de las personas es fundamental, podría ayudar a tener una mejor comprensión de lo que abarca la calidad de vida más allá de lo económico y cómo influye en nuestra vida desde el ámbito individual como colectivo. Para la presente investigación se entenderá la felicidad como un estado afectivo de satisfacción plena que experimenta subjetivamente una persona... (Alarcón, 2006).

El propósito del estudio se centra en conocer la influencia de los años de estudios en relación con la realización personal de los estudiantes universitarios de URACCAN, utilizando, como instrumento para la recopilación de la información, la escala factorial para medir la felicidad de Alarcón (2006). Los resultados obtenidos se analizaron así 
desde diferentes perspectivas, empleando el paquete estadístico SPSS y aplicando varias pruebas, como la de la normalidad de Kolmogorov, o la prueba U de Mann-Whitney, entre otros, lo que facilita determinar si variables como edad, años de estudio, etnia o carrera de los estudiantes ejercen algún tipo de influencia sobre la variable felicidad, en sus diferentes dimensiones.

\section{Literatura}

La felicidad ha tenido para los hombres y mujeres singular valor entre las metas de sus vidas. Es un tema que ha sido tratado por diferentes autores y desde diferentes perspectivas. El informe mundial de felicidad 2019 ubica a cuatro naciones nórdicas en el top ten de felicidad mundial: Noruega, Dinamarca, Suecia e Islandia, ocupando Nicaragua el puesto 45 del ranking mundial.

Y nos preguntamos, ¿Qué es la felicidad? Desde la perspectiva filosófica, Aristóteles afirma que el hombre feliz es el que vive y obra bien, porque la felicidad es una especie de vida dichosa y de conducta recta, consiste en la posesión de la sabiduría. Para ser feliz debe disponerse de bienes exteriores que permitan dedicarse a la vida contemplativa y satisfacer sus propias necesidades. Epicuro sostiene que el hombre busca la vida dichosa y placentera y que huye de todo lo que causa dolor, pena y preocupación siendo un principio esencial de la felicidad: el cuerpo humano y la mente que lo habilita (Alarcón, 2015, p. 7).

Actualmente existen diversas escalas para evaluar bienestar que se asocian a la evaluación de la felicidad a nivel teórico y en su contenido temático o en sus dimensiones, Asi tenemos, entre otros la Satisfacción con la escala de la vida (Pavot \& Diener, 1993), la Escala de Felicidad Subjetiva (Lyubomirsky y Lepper, 1999), La escala de vida de la depresión coprta (SDHS, Joseph et al., 2004), el Índice de felicidad de Steen (SHI, Seligman, et al., 2005) y la Escala factorial de felicidad (Alarcón, 2006).

En este trabajo, utilizaremos la escala de felicidad de Alarcón (2006) quien define felicidad como un estado afectivo de satisfacción plena que experimenta subjetivamente una persona y que se considera sinónimo de bienestar subjetivo, inspirada en las ideas de Aristóteles y Epicuro, y en los hallazgos recientes de la investigación empírica de la felicidad o bienestar subjetivo.

El autor reporta cuatro dimensiones o factores de la felicidad, a saber (ToribioPérez et al., 2012):

- Factor 1. Sentido positivo de la vida: Indican profunda depresión, fracaso, intranquilidad, pesimismo y vacío existencial. Las respuestas de rechazo a lo que afirman las oraciones, indican grados de felicidad, que reflejan actitudes y experiencias positivas hacia la vida. Significa estar libre de estados depresivos 
profundos, tener sentimientos positivos hacia sí mismo y hacia la vida. El factor 1 se compone de 11 ítems, explica el $32.82 \%$ de la varianza total y sus cargas factoriales son elevadas; esta sub-escala posee una alta confiabilidad ( $=0.88)$.

- Factor 2. Satisfacción con la vida: Expresa satisfacción por lo que se ha alcanzado y la persona cree que está donde debe de estar, o que se encuentra muy cerca de alcanzar el ideal de su vida. Se compone de seis ítems, y explica el $11.22 \%$ de la varianza total. Posee una alta confiabilidad $(=0.79)$.

- Factor 3. Realización personal: aquí los reactivos expresan lo que se podría llamar felicidad plena, y no estados temporales del estar feliz. Estos ítems señalan autosuficiencia, autarquía, tranquilidad emocional, placidez; todas son condiciones para conseguir el estado de felicidad completa. El concepto de realización personal guarda correspondencia con la definición de felicidad que supone la orientación del individuo hacia metas que considera valiosas para su vida. Se compone de seis ítems, explica el 10.7\% de la varianza total (=0.76).

- Factor 4. Alegría de vivir: la denominación que lleva este factor obedece a que sus ítems señalan lo maravilloso que es vivir, refieren experiencias positivas de la vida y a sentirse generalmente bien. Tiene cuatro ítems y explica el 10.7 de la varianza total $(=0.72)$.

La escala comprende correlaciones ítem-escala, encontrándose coeficientes $r$ producto-momento altamente significativas en todos los ítems $(r=.511 ; \mathrm{p}<.001)$ ( total $=0.916)$.

Finalmente, Alarcón (2006) sostiene que la felicidad se evalúa en función al estado afectivo y cognitivo, por medio del cual se evalúan aspectos positivos y negativos, emitiendo un juicio global acerca de la satisfacción con la vida, por ende, se emite una conducta feliz. Por tanto, el estudio de la felicidad de las personas podría ayudar a tener una mejor comprensión de lo que abarca la calidad de vida más allá de lo económico y cómo influye en nuestra vida desde el ámbito individual como colectivo.

\section{Metodología de investigación}

\subsection{Enfoque de la Investigación}

El enfoque de esta investigación es cuantitativo. Este paradigma ha guiado el tratamiento de los datos a través de la categorización y descripción de las propiedades, características y perfiles de las personas, grupos, comunidades, procesos y objetos que se han sometido a análisis (Hernández et al., 2010 citado por Flores, 2016, p. 35). 


\subsection{Diseño de la Investigación}

El alcance de la investigación es correlacional, porque busca evaluar el grado de asociación entre dos o más variables, midiendo cada una de ellas (aparentemente relacionadas) y, posteriormente, cuantifican y analizan la vinculación; sustentando dichas correlaciones en hipótesis sometidas a prueba (Hernández et al., 2010, p. 81).

Los estudios correlacionales buscan conocer cómo se puede comportar un concepto o una variable al conocer el comportamiento de otras variables vinculadas (Hernández et al., 2010, p. 82); en ese sentido se pretendía conocer si variables como edad, años de estudio, etnia, carrera ejercían alguna influencia sobre la variable felicidad, en sus diferentes dimensiones.

Para el proceso de investigación, se plantearon los siguientes objetivos:

1. Conocer la influencia de los años de estudios en relación con la realización personal de los estudiantes universitarios.

2. Conocer si los estudiantes universitarios han variado en su opinión sobre la Alegría de vivir antes y después de su realización personal.

3. Conocer si la edad de los estudiantes universitarios influye en las variables: sentido positivo de la vida, satisfacción con la vida, realización personal; alegría de vivir.

4. Identificar si hay diferencias en la valoración que hacen los estudiantes universitarios, sobre el sentido positivo de la vida, satisfacción con la vida y la alegría de vivir.

5. Conocer si el grupo étnico al que pertenecen los estudiantes universitarios influye en las variables: sentido positivo de la vida, satisfacción con la vida, realización personal y alegría de vivir.

\subsection{Descripción de los participantes}

El estudio se llevó a cabo con una muestra de 237 estudiantes de las carreras Licenciatura en Enfermería, Ingeniería Agroforestal, Licenciatura en Administración de Empresas, Ingeniería Civil, así como estudiantes del Doctorado en Estudios Interculturales, de la Universidad de las Regiones Autónomas de la Costa Caribe 
Nicaragüense, recinto las Minas (véase la figura 1). Del total de participantes, el 59\% (141) fueron mujeres, y el 41\% (96) hombres. Respecto a su edad, se refleja que el $75.5 \%$ de los participantes corresponde a las edades entre los 16 a los 23 años. En referencia a la variable etnia: el $82.28 \%$ son del grupo étnico mestizos, el 7.59\% indígenas Miskito; el 7.59\% indígenas Mayangna; y el 2.53\% Creole (afro-descendientes).

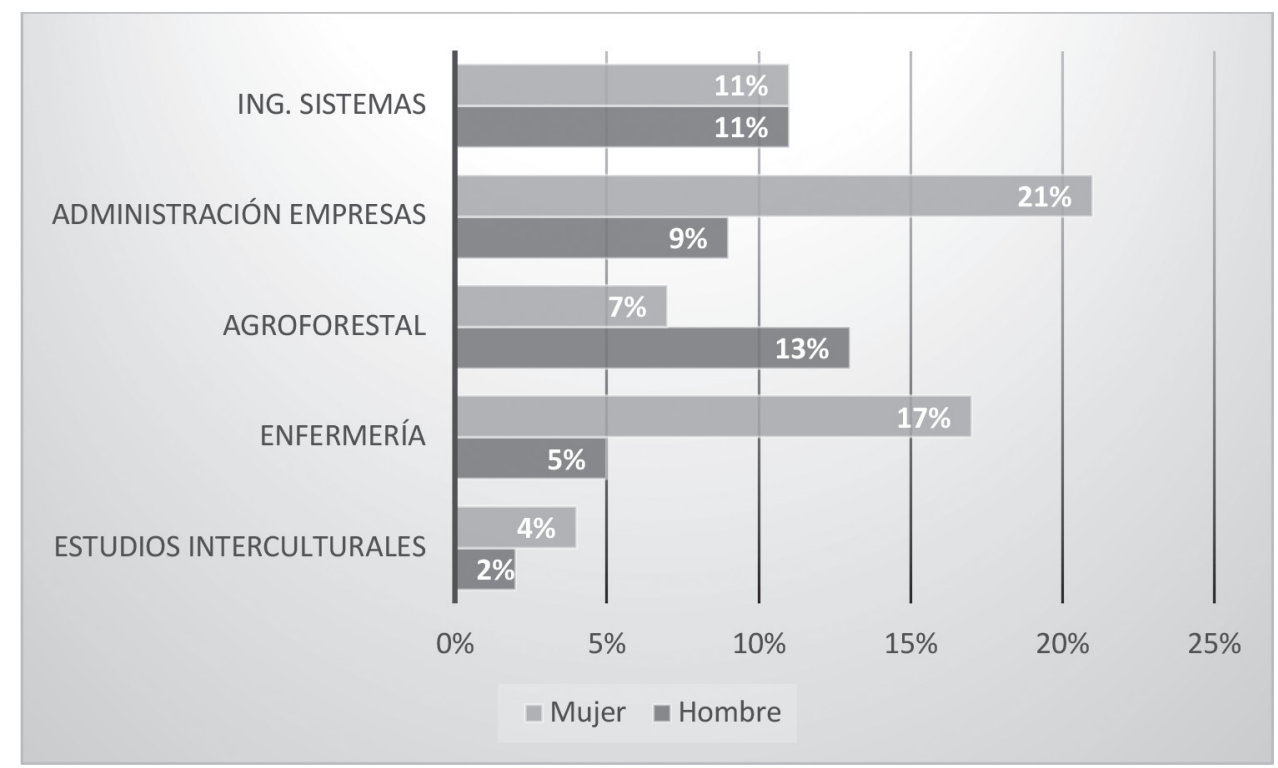

Figura 1: Distribución de género por carrera.

El tipo de muestreo implementado ha sido probabilístico, en donde se trató de que todos los elementos de la población (en este caso el total de estudiantes del recinto Las Minas), tuvieran la misma posibilidad de ser escogidos para ser parte de la muestra; para ello se definió las características de la población y el tamaño de la muestra (Hernández et al., 2010).

\subsection{Instrumento recolección de datos}

El instrumento utilizado para la recopilación de la información fue la escala factorial para medir la felicidad de Alarcón (2006). Dicho instrumento está constituido por 27 ítems, aglutinados en 4 factores asociados a la felicidad. Es una escala de tipo Likert, la cual contiene cinco valores: Fuertemente de acuerdo, de acuerdo, indiferente, no estoy de acuerdo, y fuertemente en desacuerdo. Al extremo positivo se le otorgó cinco puntos y al extremo negativo un punto. Los ítems fueron redactados en forma favorable o desfavorable a la felicidad. En el cuestionario los ítems están distribuidos aleatoriamente. Como se observa, cada ítem le daba la posibilidad a los estudiantes, 
de seleccionar la alternativa, dependiendo de su grado de acuerdo o desacuerdo. A continuación, se describen los cuatro factores y los Ítems correspondientes:

Cuadro 1: Descripción de los factores y sus Ítems

\begin{tabular}{|c|c|c|}
\hline Factor/dimensión & No. ítems & Ítems \\
\hline Sentido positivo de la vida & 11 & $\begin{array}{l}\text { 26. Todavía no he encontrado Sentido a mi existencia. } \\
\text { 20. La felicidad es para algunas personas, no para mí. } \\
\text { 18.Tengo problemas tan hondos que me quitan la tranquilidad. } \\
\text { 23. Para mí, la vida es una cadena de sufrimientos. } \\
\text { 22. Me siento triste por lo que soy. } \\
\text { 02. Siento que mi vida está vacía. } \\
\text { 19. Me siento un fracasado. } \\
\text { 17. La vida ha sido injusta conmigo. } \\
\text { 11. La mayoría del tiempo no me siento feliz. } \\
\text { 07. Pienso que nunca seré feliz. } \\
\text { 14. Me siento inútil. }\end{array}$ \\
\hline Satisfacción con la vida & 6 & $\begin{array}{l}\text { 04. Estoy satisfecho con mi vida. } \\
\text { 03. Las condiciones de mi vida son excelentes. } \\
\text { 05. La vida ha sido buena Conmigo. } \\
\text { 01. En la mayoría de las cosas, mi vida está cerca de mi ideal. } \\
\text { 06. Me siento satisfecho con lo Que soy. } \\
\text { 10. Me satisfecho porque estoy donde tengo que estar. }\end{array}$ \\
\hline Realización personal & 6 & $\begin{array}{l}\text { 24. Me considero una persona realizada. } \\
\text { 21. Estoy satisfecho con lo que hasta ahora he alcanzado. } \\
\text { 27. Creo que no me falta nada. } \\
\text { 25. Mi vida transcurre plácidamente. } \\
\text { 09. Si volviera a nacer no cambiaría casi nada de mi vida. } \\
\text { 08. Hasta ahora he conseguido las cosas que para mí son impor- } \\
\text { tantes. }\end{array}$ \\
\hline Alegría de Vivir & 4 & $\begin{array}{l}\text { 12. Es maravillo vivir. } \\
\text { 15. Soy una persona optimista } \\
\text { 16. He experimentado la alegría de vivir. } \\
\text { 13. Por lo general, me siento bien }\end{array}$ \\
\hline
\end{tabular}

Con la finalidad de garantizar la calidad de la medida, al instrumento, se le aplicó diferentes pruebas, por medio de la cual se comprobó los valores de validez y fiabilidad de este. Para ello, se calculó el valor de consistencia interna del total de los ítems del cuestionario, obteniendo un valor alfa de Cronbach del 89.5\% de confiabilidad. Con respecto a la validez, se realizó un análisis de componentes principales (ACP). La 
prueba Kaiser-Meyer-Olkin arrojo una puntuación de o,884. Por su parte, la prueba de esfericidad de Bartlett también ofreció resultados que indicaban que el análisis era pertinente (Chi-cuadrado=2129.646; g.l. $=351 ; \mathrm{p}<0,000)$. De igual manera se calculó el determinante de la matriz de correlaciones, cuyo valor fue ooooo $8.139(\mathrm{D}=8.139-5)$, cuyo valor fue menor que o, por tanto, es un instrumento válido.

\subsection{Procedimientos de administración y enfoque ético}

La aplicación del instrumento fue realizada por los autores, durante el segundo semestre del curso académico 2019. Fue administrado de manera colectiva dentro de las aulas de clase, de forma anónima y en presencia de los docentes. Con anterioridad a la toma de los datos, se obtuvo el consentimiento previo, libre e informado del estudiantado, docentes, así como el permiso de las autoridades universitarias.

\subsection{Análisis de datos}

Para alcanzar el objetivo del estudio se procedió al desarrollo de distintos análisis, empleando el paquete estadístico SPSS v.24. Entre ellos, se aplicó el análisis de la consistencia interna del cuestionario, el análisis de componentes principales, y un estudio de correlaciones entre las categorías, para ello se aplicó, la prueba de normalidad de Kolmogorov, la prueba U de Mann-Whitney para dos muestras independientes, prueba de Wilcoxon, para dos muestras relacionadas, Kruskal Wallis, para muestras independientes y la prueba de Friedman para muestras relacionadas.

\section{Resultados}

\subsection{Un estudio no paramétrico de la Escala de la Felicidad}

La escala de la felicidad aplicada al estudiantado tiene cuatro dimensiones: Sentido positivo de la Vida, satisfacción con la vida, realización personal y alegría de vivir. Estas dimensiones vienen a explicar los 27 ítems que conforma la escala de la felicidad.

Con el fin de poder realizar un análisis correlacional entre las dimensiones, se procedió a realizar la prueba de Kolmogorov-Smirnov, que describe el ajuste de una distribución teórica a la distribución normal. Los resultados obtenidos nos permiten realizar el análisis de las dimensiones mediante pruebas paramétricas o bien pruebas no paramétricas, según datos obtenidos.

Tabla 1: Prueba de Kolmogorov-Smirnov para una muestra

\begin{tabular}{|c|l|l|l|l|l|}
\hline & $\begin{array}{c}\text { Sentido } \\
\text { Positivo/Vida }\end{array}$ & $\begin{array}{c}\text { Satisfacción } \\
\text { Vida }\end{array}$ & $\begin{array}{c}\text { Realización } \\
\text { Personal }\end{array}$ & $\begin{array}{c}\text { Alegría } \\
\text { Vivir }\end{array}$ & Felicidad \\
\hline N & 237 & 237 & 237 & 237 & 237 \\
\hline
\end{tabular}

92 I REVISTA ELECTRÓNICA DE CONOCIMIENTOS, SABERES Y PRÁCTICAS, Volúmen 2, Año 2, No. 2, Julio-Diciembre, 2019. 


\begin{tabular}{|c|c|c|c|c|c|c|}
\hline \multirow[t]{2}{*}{ Parámetros normales ${ }^{\mathrm{a}, \mathrm{b}}$} & Media & 42,1055 & 23,0295 & 20,3249 & 15,316 & 100,77 \\
\hline & $\begin{array}{l}\text { Desviación } \\
\text { estándar }\end{array}$ & 8,35504 & 4,28576 & 4,40530 & 3,0163 & 16,07 \\
\hline \multirow{3}{*}{$\begin{array}{l}\text { Máximas diferencias } \\
\text { extremas }\end{array}$} & Absoluta &, 085 & ,138 & ,091 & ,138 & ,085 \\
\hline & Positivo & 061 & ,068 & ,055 & ,069 & ,044 \\
\hline & Negativo &,- 085 &,- 138 &,- 091 &,- 138 &,- 085 \\
\hline \multicolumn{2}{|l|}{ Estadístico de prueba } & ,085 & ,138 & ,091 & ,138 & ,085 \\
\hline \multicolumn{2}{|l|}{ Sig. asintótica (bilateral) } &, $000 \mathrm{C}$ &, $000 C$ &, $000 C$ &, $000 C$ &, $000 C$ \\
\hline \multicolumn{7}{|c|}{ a. La distribución de prueba es normal. } \\
\hline \multicolumn{7}{|c|}{ b. Se calcula a partir de datos. } \\
\hline \multicolumn{7}{|c|}{ c. Corrección de significación de Lilliefors. } \\
\hline
\end{tabular}

Los resultados obtenidos de la prueba de normalidad (Kolmogorov-Smirnov), para las variables descritas muestran que el nivel de significancia obtenido para cada dimensión es menor que o,05, lo cual viene a rechazar la normalidad de las dimensiones analizadas. Por tanto, se concluye que las puntuaciones obtenidas en la felicidad y sus dimensiones, no tiene el comportamiento de una distribución normal, lo cual sugiere realizar pruebas no paramétricas para analizar el comportamiento correlacionar de las dimensiones con las variables de la escala de la Felicidad.

\subsection{Influencia de los años de estudio en relación con la realización personal de los estudiantes universitarios}

Para conocer la influencia de los años de estudio en relación con la realización personal de los estudiantes universitarios, se realizó la prueba de U de Mann-Whitney, que es una prueba no paramétrica aplicada para muestras independientes. Para ello, se realizó una prueba de hipótesis, la cual verifica si los años de estudios de los universitarios, tienen una influencia con relación a la dimensión de la realización personal, la cual se describe a continuación:

a. $H_{o}$ : Los años de estudios no influyen en la realización personal de los estudiantes universitarios.

b. $H_{1}$ : Los años de estudios influyen en la realización personal de los estudiantes universitarios.

Se procedió a realizar la prueba U de Mann-Whitney y se obtuvieron los siguientes resultados. 
Tabla 2: Prueba U de Mann-Whitney de dos muestras independientes

\begin{tabular}{|l|l|}
\hline & \multicolumn{1}{|c|}{ Realización Personal } \\
\hline U de Mann-Whitney & 5657,500 \\
\hline W de Wilcoxon & 9662,500 \\
\hline Z & $-1,821$ \\
\hline Sig. asintótica (bilateral) &, 069 \\
\hline a. Variable de agrupación: Año de Estudio & \\
\hline
\end{tabular}

Basado en la prueba de Mann-Whitney se demuestra que p>0,05, por tanto no existen diferencias estadísticamente significativas. Se rechaza la hipótesis nula, es decir, los años de estudios no influyen en la realización personal porque el nivel de significancia es mayor (Sig. 0,069).

\subsection{Variación de opinión sobre la alegría por vivir antes y después de su realización personal}

Con el fin de conocer si la percepción de la alegría de vivir depende de la realización personal del estudiantado universitario, se realizó la prueba de Wilcoxon, que compara el rango medio de dos muestras relacionadas, y de esta manera se podría determinar si existen diferencias entre ellas. Para ello, se planteó las siguientes hipótesis:

a. $H_{o}$ : No hay diferencias en la opinión de los estudiantes universitarios sobre la alegría de vivir entre antes y después de su realización personal.

b. $H_{1}$ : Hay diferencias en la opinión de los estudiantes universitarios sobre la alegría de vivir entre antes y después de su realización personal.

Tabla 3: Estadístico de prueba de Wilcoxon de dos muestras relacionadas

\begin{tabular}{|l|l|}
\hline & Realización Personal - Alegría de vivir \\
\hline$Z$ & $-12,529^{\mathrm{b}}$ \\
\hline Sig. asintótica (bilateral) &, 000 \\
\hline a. Prueba de rangos con signo de Wilcoxon \\
\hline b. Se basa en rangos negativos. & \\
\hline
\end{tabular}

Basado en la prueba de Wilcoxon se rechaza la hipótesis nula porqué existen diferencias significativas entre las variables $(p<0,05)$. Se puede concluir que hay diferencias en la valoración de los estudiantes sobre la alegría de vivir antes y después de su realización personal. 


\subsection{Influencia de la edad de los estudiantes universitarios en las variables: sentido positivo de la vida, satisfacción con la vida, realización personal y alegría de vivir.}

Para conocer si la edad de los estudiantes universitarios influye en las variables: sentido positivo de la vida, satisfacción con la vida, realización personal, alegría de vivir, se realizó la prueba de Kruskal Wallis con las siguientes hipótesis:

\section{Hipótesis 1:}

a. $H_{o}$ : La edad de los estudiantes universitarios no influyen en los factores asociados a la felicidad.

b. $H_{1}$ : La edad de los estudiantes universitarios influyen en los factores asociados a la felicidad.

Tabla 4: Estadístico de prueba Kruskal Wallis de k muestras independientes

\begin{tabular}{|l|l|l|l|l|}
\hline & \multicolumn{1}{|c|}{$\begin{array}{c}\text { Sentido Positivo de } \\
\text { la Vida }\end{array}$} & \multicolumn{1}{|c|}{$\begin{array}{c}\text { Satisfacción con la } \\
\text { vida }\end{array}$} & Realización Personal & \multicolumn{1}{|c|}{ Alegría de vivir } \\
\hline Chi-cuadrado & 3,526 & 2,358 & 4,927 & 2,091 \\
\hline gl & 2 & 2 & 2 & 2 \\
\hline Sig. asintótica &, 172 &, 308 &, 085 & 351 \\
\hline a. Prueba de Kruskal Wallis \\
\hline b. Variable de agrupación: Edad de la Persona \\
\hline
\end{tabular}

Basado en la prueba Kruskal Wallis de k muestras independientes se demostró que no existen diferencias estadísticamente significativas. La edad de los estudiantes no influye en los factores asociados a la felicidad, porque el valor de p>0,05. Por tanto, se rechaza la hipótesis alternativa.

\subsection{Valoración que hacen los estudiantes universitarios, sobre el sentido positivo de la vida, satisfacción con la vida y la alegría de vivir.}

Para poder identificar si hay diferencias en la valoración que hacen los estudiantes universitarios sobre el sentido positivo de la vida, satisfacción con la vida y alegría de vivir, se procedió a realizar la prueba de Friedman para k muestras relacionadas. Para ello, se plantearon las siguientes hipótesis:

a. $H_{o}$ : No hay diferencias de valoración entre el sentido positivo de la vida; satisfacción con la vida y alegría de vivir 
b. $H_{1}$ : Sí hay diferencias de valoración entre el sentido positivo de la vida; satisfacción con la vida y alegría de vida

Tabla 5: Estadístico de prueba de Friedman de k muestras relacionadas

\begin{tabular}{|l|l|}
\hline $\mathrm{N}$ & 237 \\
\hline Chi-cuadrado & 454,711 \\
\hline $\mathrm{gl}$ & 2 \\
\hline Sig. asintótica &, 000 \\
\hline a. Prueba de Friedman & \\
\hline
\end{tabular}

Basado en la prueba de Friedman para muestras relacionadas, se acepta la hipótesis alternativa y se concluye que existen diferencias estadísticamente significativas de la percepción de los estudiantes en relación con las tres variables, con un valor de significancia menor que 0,05 .

\subsection{Influencia de la variable etnia de los estudiantes en los factores de la} felicidad: sentido positivo de la vida, satisfacción con la vida, realización personal y alegría de vivir.

Para conocer si el grupo étnico influye en las variables, se utilizó la prueba de Kuskall Wallis para k-muestras independientes y se plantearon las siguientes hipótesis:

\section{Hipótesis 1:}

c. $H_{o}$ : La etnia de los estudiantes universitarios no influyen en los factores asociados a la felicidad.

d. $H_{1}$ : La etnia de los estudiantes universitarios influyen en los factores asociados a la felicidad.

Tabla 6: Estadístico de prueba Kruskal Wallis para k muestras independientes

\begin{tabular}{|l|l|l|l|l|}
\hline & $\begin{array}{c}\text { Sentido Positivo } \\
\text { de la Vida }\end{array}$ & $\begin{array}{l}\text { Satisfacción con } \\
\text { la vida }\end{array}$ & Realización Personal & Alegría de vivir \\
\hline Chi-cuadrado & 4,796 &, 611 & 1,635 & 5,437 \\
\hline gl & 2 & 2 & 2 & 2 \\
\hline Sig. asintótica &, 091 &, 737 &, 442 &, 066 \\
\hline a. Prueba de Kruskal Wallis \\
\hline \multicolumn{5}{|l}{ c. Variable de agrupación: Etnia de la persona } \\
\hline
\end{tabular}

Basado en la prueba de Kruskall Wallis, se observa que todos los grupos étnicos tienen la misma percepción sobre los factores asociados a la felicidad, porque el valor de la significancia es mayor al o,05. Por tanto se acepta la hipótesis nula. 


\section{Discusión y conclusiones}

La felicidad de una persona está asociada y entendida a la forma en que una persona se siente, otros lo asocian con la satisfacción de los logros obtenidos en la vida y falta de ocurrencia de eventos que viene afectar la forma de vivir de una persona. La escala de felicidad aplicada al estudiantado de la universidad URACCAN-recinto Las Minas, recoge los elementos descritos en estas concepciones de la felicidad.

Esta investigación trata de responder a la pregunta ¿es posible la felicidad en estudiantes universitarios?, la escala de la felicidad compuesta por cuatro factores, fue aplicada a estudiantes universitarios, considerando las variables género, edad, etnia y años de estudio encontrándose lo siguiente:

La prueba de Mann-Whitney aplicada a la dimensión de la realización personal con relación a los años de estudio de los universitarios, considera que esta última no influye sobre la realización personal. Esto indica que, para él universitario, su realización personal está asociada a otros factores, coincidiendo con Alarcón (2006).

Por otro lado, la prueba de Wilcoxon de dos muestras relacionada, concluye que hay diferencias en la valoración de los estudiantes sobre la alegría de vivir antes y después de la realización personal. Es decir, el estudiante se considera alegre antes de su realización personal.

Por otro lado, la prueba de Friedman demostró que existen diferencias estadísticamente significativas de la percepción de los estudiantes en relación al sentido positivo, satisfacción por la vida y alegría de vivir, expresando no tener la misma percepción de las dimensiones de la felicidad.

$\mathrm{Al}$ analizar la relación de la variable edad del estudiantado, con los factores de la felicidad, la prueba de Kruskal-Wallis, indican que la edad de los universitarios no influye en las dimensiones asociadas a la felicidad.

Por otro lado, el Estadístico de Prueba de Kruskal-Wallis para k-muestras independiente refleja que todos los grupos étnicos tienen la misma percepción sobre la felicidad. Este resultado, refleja las mismas condiciones, realidades similares en que se encuentran las edades y los grupos étnicos en la universidad, por lo que la percepción de la felicidad es la misma en la universidad.

Este estudio nos ha permitido tener una mejor comprensión de lo que es felicidad, más allá de lo económico, así como los elementos vinculados a la felicidad, los cuales inciden en el ámbito individual y colectivo de una persona; tal y como lo menciona Alarcón (2006). 
Podemos concluir que la felicidad en estudiantes universitarios sí es posible, tomando en cuenta que la misma es meramente subjetiva en las personas y a su alrededor se engloban diferentes elementos que la hacen posible. La etnia, edad, género y años de estudio no tienen una relación directa con el estado de felicidad de los estudiantes, pues no son determinantes para definir este estado en el ser humano. Por tanto se sugiere profundizar el estudio considerando variables centradas en las emociones o sea en el "ser y los sentires" de los estudiantes.

\section{Agradecimiento}

Nuestro agradecimiento a la Universidad de la Regiones Autónomas de la Costa Caribe Nicaragüense, URACCAN y al proyecto "Dialogo de saberes y haceres fortaleciendo el ejercicio de la autonomía" auspiciado por SAIH, por el financiamiento de esta investigación.

\section{Lista de referencias}

Alarcón, Reynaldo (2002). Fuentes de la felicidad: ¿Qué hace feliz a la gente? Universidad Ricardo Palma. Revista de Psicología de la PUCP. 20(2), 12-17.

Alarcón, Reynaldo (2006). Desarrollo de una Escala Factorial para Medir la Felicidad. Revista Interamericana de Psicología/Interamerican Journal of Psychology, 40(1), 96-106.

Alarcón, Reynaldo (2015). La idea de la felicidad. Universidad Ricardo Palma. Apuntes Ciencias Sociales, 5(1).

Flores W. (2016). Análisis ontosemiótico en los procesos de resolución de problemas matemáticos por estudiantes Universitarios. Bilbao: Universidad Deusto.

Helliwell, J., Layard, R., \& Sachs, J. (2019). World Happiness Report 2019. New York: Sustainable Development Solution Network. New York, NY, USA. Page 24.

Hernández R., Fernández C., Baptista P. (2010). Metodología de la Investigación. México: Editorial McGraw Hill

Toribio-Pérez, L., Gonzáles-Arratia, N., Valdez Medina, J., Gonzales Escobar, S., Van Barneveld, H. (2012). Validación de la escala de la felicidad de Alarcón para adolescentes mexicanos. Psicología Iberoamericana, 1, 71-79.

Vera-Villaroel, Pablo; Celis-Atenas, Karem y Cordova-Rubio, Natalia (2011). Evaluación de la Felicidad: Análisis Psicométrico de la Escala de Felicidad Subjetiva en Población Chilena. 29(1), 127-133. 